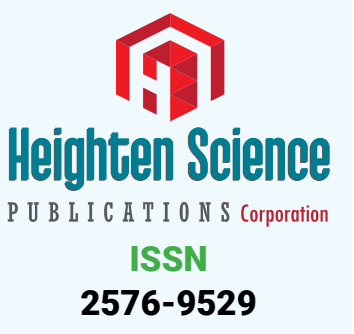

2576-9529
*Address for Correspondence: Doaa M Youssef, Professor of Pediatrics, Zagazig University, Egypt, Tel: +201222839220;

Email: dody5176@yahoo.com

Submitted: 02 May 2019

Approved: 30 May 2019

Published: 31 May 2019

Copyright: @ 2019 Abu Zeid AM, et al. This is an open access article distributed under the Creative Commons Attribution License, which permits unrestricted use, distribution, and reproduction in any medium, provided the original work is properly cited

Keywords: Acute kidney injury; Urine neutrophil gelatinase associated lipocalin; Critically ill children; Renal angina index

Check for updates
Research Article

\section{Urinary NGAL incorporation into Renal Angina Index for early detection of acute kidney injury in critically ill children}

\author{
Ali Mohammed Abu Zeid ${ }^{1}$, Doaa Youssef Mohammed ${ }^{1 *}$, \\ Amal Saeed AbdAlazeem², Anas Saad Elsayed mohammed \\ Seddeeq $^{3}$ and Ashraf Mohamed Elnaany ${ }^{4}$ \\ 'Professor of Pediatric, Zagazig Universiy, Egypt \\ ${ }^{2}$ Assistant Professor of Biochemistry, Zagazig University, Egypt \\ ${ }^{3}$ Resident of Neonatology, Zagazig University, Egypt \\ ${ }^{4}$ Assistant Professor of Urology, Benha University, Egypt
}

\section{Abstract}

Background and objectives: New AKI biomarkers (on the top of it NGAL biomarker) have demonstrated better performance for prediction of AKI in critically ill patients with heterogeneous illness. Renal angina index was recently reported to enhance prediction of severe AKI at the time of intensive care unit admission. This study tested the hypothesis that incorporation of UNGAL in patients with renal angina improves the prediction of severe AKI.

Design, setting, participants \& measurements: In our study 53critically ill children admitted to the pediatric intensive care unit in Zagazig university hospital, Measurement of urine neutrophil gelatinaseassociated lipocalin (UNGAL) was determined individually by ELISA kit and in combination with the RAI which is calculated in each critically ill child for severe AKI. Statistical analysis was done for these data.

Results: Individual uNGAL demonstrated marginal discrimination for severe AKI (area under curve [AUC]: NGAL, 0.877), little higher than prediction by RAI (AUC $=0.847$ ). Incorporation of UNGAL significantly added to the renal angina index AKI prediction (AUC=0.847, increased to 0.893 ).

Conclusion: This study shows that incorporation of UNGAL into the RAI improves detection ability of severe AKI in critically ill children.

\section{Introduction}

Acute kidney injury (AKI) occurs frequently in critically ill patients and is associated with high rates of morbidity and mortality. Recently over 3.5 million patients, the incidence of AKI was $21.6 \%$ in adults and $33.7 \%$ in children, with mortality rates of 23.9 and $13.8 \%$, respectively [1]. Since the adoption of severity-based classifications of injury, there has been an increasing awareness of the danger of AKI. Unfortunately, no singular effective therapy for AKI has been developed and management consists largely of supportive care [2]. The Kidney Diseases Improving Global Outcomes (KDIGO) working group recently described a stage-based best practice AKI management algorithm, notably highlighting recommendations after recognition of a patient with subclinical AKI or AKI risk [3]. Prediction of AKI offers the opportunity to initiate measures for AKI prevention. Creatinine-based detection strategies, limited in efficacy by time-lag in response to injury and variability with body mass and gender,

How to cite this article: Abu Zeid AM, Mohammed DY, AbdAlazeem AS, Mohammed Seddeeq ASE Elnaany AM. Urinary NGAL incorporation into Renal Angina Index for early detection of acute kidney injury in critically ill children. J Clini Nephrol. 2019; 3: 093-099. https://doi.org/10.29328/journal.jcn.1001032 
are particularly problematic in the pediatric population [4]. New biomarkers have demonstrated better performance for the prediction of AKI compared with early nonstratified changes in serum creatinine ( $\mathrm{SCr}$ ). Validated following cardiopulmonary bypass (CPB) in children, urinary neutrophil gelatinase-associated lipocalin (uNGAL), detect AKI before the functional change deduced by S.Creatinine rise [5]. The renal angina index (RAI) is a composite of patient AKI risk and early signs of injury. In short, fulfillment of renal angina informs the pretest probability of any AKI diagnostic test [6].

\section{Patient and Method}

\section{Population of the study}

53 Critically ill children aged from 3 months to 7 years 85\% (45/53) of them males were enrolled for this study, all were used to extract demographic and outcome data. We used these data for day $3 \mathrm{AKI}$ analysis, we measured NGAL in urine for all children at the day of admission. 16 critically ill children were bronchopneumonia, 5 children were ATN, 10 children were dehydration and 19 children were sepsis. 25 critically ill children were RAI+ with the first 12 hours in PICU; these children had been associated with poor outcome. Day 3 AKI incidence was 18.9\% (10/53); 90\% (9/10) of day 0 RAI+ were AKI+ on day3,50\%(5/10) of AKI underwent for RRT two underwent peritoneal dialysis and three received intermittent acute hemodialysis of these three patients two had nephrostomy as well, two (2) children were discharged under supportive treatment the other three children became chronic kidney disease.

\section{Sample size calculation}

A sample size of 53 students was obtained by using Epi Info software version 7.1.4.0, with power $80 \%, 95 \%$ confidence level and 5\% marginal error.

\section{Sampling technique}

\section{Data Timing.}

- Data was collected at (Day 0,3,7) from PICU admission.

- A minimum of $8 \mathrm{~h}$ from PICU admission considered Day 0 .

- Urinary NGAL was assessed the day after PICU admission, between 12 and 24h after time of admission (Day 1).

- Day 3 is the time period between 72 and $96 \mathrm{~h}$ after PICU admission.

2. Collected variables.

- Demographic information, admission diagnoses, comorbidities, height, weight, available laboratory values and vital signs were collected at the time of admission.

- Baseline SCr was established as the lowest creatinine up to 3 months before PICU admission on Day 0, If no baseline S.Creatinin is available in the computer system, a reference estimated creatinine clearance of $120 \mathrm{~mL} / \mathrm{min} / \mathrm{m} 2$ was used and a baseline creatinine was imputed using the patient's height in centimeters (schwartz method) [( $\mathrm{crcl}=\mathrm{k}^{*} \mathrm{Ht} / \mathrm{s} . \mathrm{cr}$ ) $\mathrm{k}=0.45$ in infant, $\mathrm{k}=0.55$ in child] [7].

- Daily collected variables was assessed at 8:00 am for each patient on (Days $0,3,7)$ and included vital signs, laboratory values, nephrotoxin use, vasopressor use, mechanical ventilation support level and total ICU fluid intake and output.

3. Calculated variables and RAI. 
- The RAI score was determined between 8 and $12 \mathrm{~h}$ from the time of PICU admission on Day 0 as previously described at the introduction [8].

- An RAI $\geq 8$ was considered fulfillment of renal angina. Fulfillment or the absence of renal angina was denoting 'RA+' or 'RA-'. Net ICU fluid balance was determined using simple subtraction of total fluid balance during ICU course. Urine output was calculated as $\mathrm{mL} / \mathrm{kg} / \mathrm{h}$ at (day 0,3 , and 7) [8].

\section{Urine collection and biomarker analysis.}

Urine samples were collected on all enrolled patients. Urine was centrifuged and stored in aliquots at $-80^{\circ} \mathrm{C}$ until measurement. Day1 NGAL values were used for the purposes of this initial analysis (urine obtained from Days 1-4 when possible). uNGAL was assayed using a human-specific commercially available enzyme linked immunosorbent assay [8].

\section{Estimation of urinary Neutrophil Gelatinase-Associated Lipocalin (NGAL)}

Five milliliters of urine was obtained, spun at $3000 \mathrm{rpm}$ for 15 minutes. The supernatant was aliquoted equally into cryovials and stored at $-80^{\circ} \mathrm{C}$ until assay. The neutrophil gelatinase-associated lipocalin (NGAL) level in urine was assessed by an NGAL enzyme-linked immunosorbent assay kit (Kit 201-12-1720, sunred bio, shanghai, China), which specifically detects human NGAL. The assay was performed according to the manufacturer's protocol: $100 \mu \mathrm{L}$ of NGAL standards or diluted samples of urine were applied to the precoated microwells in duplicates. Microwells were then incubated for 1 hour at room temperature and then washed with washing buffer. In succession, biotinylated NGAL antibody and horseradish peroxidase-streptavidin were incubated in the wells for 1 hour each with shaking. Tetramethylbenzidine dihydrochloride substrate was added for 10 minutes in the dark before adding stop solution. Finally, NGAL concentration was measured at $450 \mathrm{~nm}$ wavelength. The absorbance was proportional to the concentration of NGAL. A standard curve was constructed by plotting absorbance value versus NGAL concentration of standards, and concentrations of unknown samples are determined using this standard curve. The intra assay coefficients of variation were $2.1 \%$ (range, $1.3 \%$ to $4.0 \%$ ). The inter assay variation was $9.1 \%$ (range, $6.8 \%$ to $18.1 \%$ ). Urine NGAL excretion is presented as the amount of urine. The urine NGAL levels were expressed as (ng/ml) [9].

\section{Data analysis}

Were analyzed using SPSS, version 16. Variables were presented as number and per cent. Chi squared test was used for comparison between groups. Odds ratio (OR) and their 95\% confidence intervals (CI) were calculated. $P \leq 0.05$ was considered significant

\section{Results}

\section{Group characteristic}

Demographics are shown in table 1 . The incidence of severe AKI at 3 days was $18.8 \%$ (10 of 53) in all critically ill children, although the incidence was significantly higher in patients with RAI >8 [ANG (+)] on admission (9 of 25 [36\%] versus 1 of 28 [3.6\%]; $\mathrm{P}=0.008$ ) (Table 2). There was no statistically significant difference in demographic data regarding RAI (Age, sex, height, weight) with day 3 AKI in critically ill children (Table 1) .

\section{NGAL biomarker}

Are little higher than the RAI for Prediction of Severe AKI. The comparison of AUC point estimates taken on day 0 showed that UNGAL biomarker demonstrated a good performance for prediction of day 3 AKI (Table 3, Figure 1). 


\section{Incorporation of uNGAL biomarker}

Augments the Predictive Performance of the RAI. The probability of AKI was calculated by determining the rate of AKI of biomarker and RAI combination, demonstrating a marked increase after incorporation of uNGAL biomarker (Table 4, Figure 2). Improvement in RAI predictive performance was greatest when NGAL was added (AUC $=0.847$, increased to 0.893).

\section{Day 3-AKI is associated with poor outcome in a heterogeneous population of critically ill children}

Day 3-AKI was associated with ICU morbidity. Day 3-AKI increased duration of ICU LOS, prolonged mechanical ventilation and was associated with a greater number of organ failure days than the absence of AKI. Day 3 AKI incidence was 18.9\% (10/53); $90 \%(9 / 10)$ of day $0 \mathrm{RAI}+$ were AKI+ on day 3 (Table 2, Figures 3-5) $50 \%(5 / 10)$ of AKI underwent for hemodialysis, two (2) children were discharged under supportive $\mathrm{ttt}$ the other three children became chronic kidney disease.

\section{Discussion}

Basu RK et al. 2014 [8], validate the advantage of the RAI methodology for AKI risk stratification RAI assessment is early (day of admission), easy to practice (calculating RAI and measuring biomarkers is relatively simple compared with calculation of severity of illness scores), useful (high negative predictive value) (Table 4), and could potentially change initial resuscitation targets (fluid goals), use of medications (nephrotoxins), and timing of adjunctive therapies (RRT).

\begin{tabular}{|c|c|c|c|c|c|c|c|}
\hline & & \multicolumn{2}{|c|}{ RAl- (No.=28) } & \multicolumn{2}{|c|}{ RAl+ (No.=25) } & \multicolumn{2}{|c|}{ Chi square test } \\
\hline & & No. & $\%$ & No. & $\%$ & $\mathrm{X}^{2} / \mathrm{t}^{*}$ & P-value \\
\hline \multirow{2}{*}{ Sex } & Female & 3 & $10.7 \%$ & 5 & $20 \%$ & \multirow{2}{*}{0.889} & \multirow{2}{*}{0.346} \\
\hline & Male & 25 & $89.3 \%$ & 20 & $80.0 \%$ & & \\
\hline Age & Mean $\pm S D$ & 2.02 & 1.95 & 2.22 & 2.01 & -0.369 & 0.713 \\
\hline Weight (kg) & Mean $\pm S D$ & 10.0 & 3.7 & 10.0 & 4.7 & 0.026 & 0.980 \\
\hline Height (cm) & Mean $\pm S D$ & 74.4 & 10.1 & 74.2 & 14.9 & 0.074 & 0.942 \\
\hline
\end{tabular}

Table 2: Comparison between RAI as regards RIFLE criteria of AKI.

\begin{tabular}{|c|c|c|c|c|c|c|c|}
\hline \multirow{2}{*}{\multicolumn{2}{|c|}{ RIFLE }} & \multicolumn{2}{|c|}{ RAl- (No.=28) } & \multicolumn{2}{|c|}{ RAl+ (No.=25) } & \multicolumn{2}{|c|}{ Chi square test } \\
\hline & & No. & $\%$ & No. & $\%$ & $\mathrm{X}^{2}$ & P-value \\
\hline \multirow{2}{*}{ DO } & $\mathrm{R}$ & 9 & $32,1 \%$ & 14 & $56,0 \%$ & \multirow{2}{*}{15.132} & \multirow{2}{*}{0.001} \\
\hline & 1 & 0 & $0,0 \%$ & 6 & $24,0 \%$ & & \\
\hline \multirow{3}{*}{ D3 } & $\mathrm{R}$ & 27 & $96,4 \%$ & 16 & $64,0 \%$ & \multirow{3}{*}{9.575} & \multirow{3}{*}{0.008} \\
\hline & 1 & 1 & $3,6 \%$ & 3 & $12,0 \%$ & & \\
\hline & $\mathrm{F}$ & 0 & $0,0 \%$ & 6 & $24,0 \%$ & & \\
\hline \multirow{5}{*}{ D7 } & $\mathrm{R}$ & 4 & $14,3 \%$ & 12 & $48,0 \%$ & \multirow{5}{*}{21.700} & \multirow{5}{*}{0.001} \\
\hline & 1 & 0 & $0,0 \%$ & 2 & $8,0 \%$ & & \\
\hline & $\mathrm{F}$ & 0 & $0,0 \%$ & 3 & $12,0 \%$ & & \\
\hline & $\mathrm{L}$ & 0 & $0,0 \%$ & 1 & $4,0 \%$ & & \\
\hline & $E$ & 0 & $0,0 \%$ & 1 & $4,0 \%$ & & \\
\hline
\end{tabular}

This table shows that there was statistically significant increase in RIFLE stages with RAI+ in comparison with RAl- at D0, D3 and D7.

Table 3: Comparison between RAl as regards NGAL.

\begin{tabular}{|l|c|c|c|c|c|c|}
\hline & \multicolumn{2}{|c}{ RAI-(No.=28) } & \multicolumn{2}{c}{ RAl+(No.=25) } & \multicolumn{2}{c|}{ Independent t-test } \\
\cline { 2 - 8 } & Mean & SD & Mean & SD & t & P-value \\
\hline NGAL $(\mathrm{ng} / \mathrm{ml})$ & 256.36 & 77.53 & 346.96 & 81.82 & -4.125 & $<0.001$ \\
\hline
\end{tabular}

This table shows that there was statistically significant increase in RAl+ in comparison to RAl- with NGAL. 
Table 4: Cut of point of RAI, NGAL and RAI+NGAL as regards AKI.

\begin{tabular}{|c|c|c|c|}
\hline & RAI & NGAL & RAI+NGAL \\
\hline Cut of point & $\geq 8$ & $\geq 323$ & $\geq 334$ \\
\hline Sensitivity & $79.23(48.2-85.7)$ & $88.46(69.8-97.6)$ & $89.46(72.8-98.6)$ \\
\hline Specificity & $74.07(53.7-88.9)$ & $66.67(46.0-83.5)$ & $78.67(63.0-93.5)$ \\
\hline PPV & $72.0(50.6-87.9)$ & $71.9(53.3-86.3)$ & $73.9(53.3-86.3)$ \\
\hline NPV & $91.4(73.3-98.7)$ & $85.7(63.7-97.0)$ & $93.7(74.7-99.5)$ \\
\hline AUC & 0.847 & 0.877 & 0.893 \\
\hline
\end{tabular}

Figure 1: NGAL regarding RAl- and RAl+.

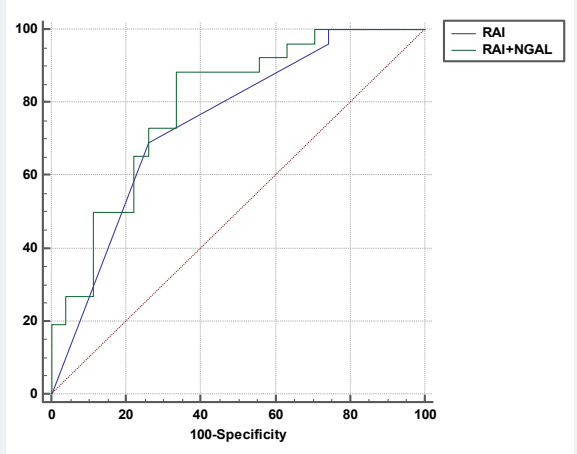

Figure 2: Multi ROC carve of RAI and RAI+NGAL in RAI- and RAI+.

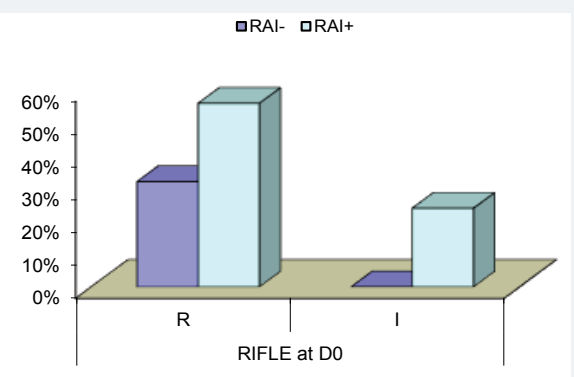

Figure 3: RIFLE (D0) regarding RAI- and RAI+

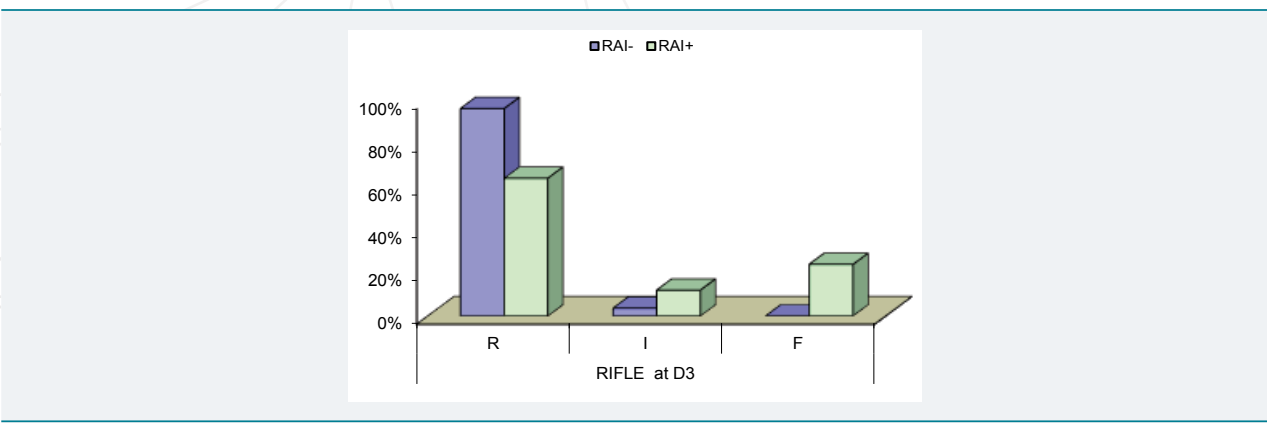

Figure 4: RIFLE (D3) regarding RAI- and RAI+. 
口RAI- QRAI+

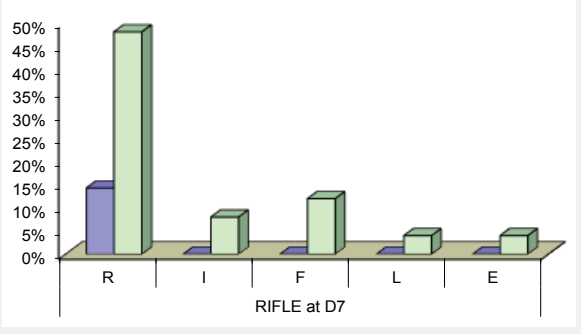

Figure 5: RIFLE (D7) regarding RAl- and RAI+.

This prospective cohort study include 53 critically ill children, an informed consent were obtained from children parents. All children included in this study underwent detailed history taking, thorough clinical examination, laboratory investigation D0 counting of RAI and measurement of UNGAL by ELISA kit.

In this study, we confirm the hypothesis that incorporation of uNGAL into RAI improves predictive ability of acute kidney injury in critically ill children and incorporation of UNGAL potentiate the predictive performance of RAI (AUC of RAI 0.847 and AUC of both RAI and NGAL is 0.893).

Our results was agreed with study of Basu RK et al. 2014 [8], which state that inclusion of ngal increase the predictive performance of RAI (AUC of RAI 0.80 and AUC of both RAI and NGAL 0.85 ).

Also our results was agreed with study of Shina Menon et al. 2016 [10], which stated that addition of NGAL to RAI enhance the predictive ability of RAI (AUC of RAI is 0.80 and AUC of both RAI and NGAL 0.97).

In our study there was statistically significant increase in day $3 \mathrm{AKI}$ according to RIFLE classification of AKI with RAI + vs. RAI- (36\% vs. 3.6\%, p-value 0.008).

Our results was agreed with study of Basu RK et al. 2014 [8], which state there was high significant increase incidence of day $3 \mathrm{AKI}$ in patients with RAI+ on day of admission (18.6\% vs. $2.9 \%$, p-value $=0.003$ ).

Also our results was agreed with study of Shina Menon et al., 2016 [10], which stated that the incidence of AKI at day 3 was high in patients with RAI + on day $0 \mathrm{vs}$. RAI- (23.1\% vs. 2.9, p-value < 0.001).

In our study there was statistically significant increase in RAI+ in comparison to RAI- with NGAL. The concentration of NGAL in urine increase with RAI+ and decrease with RAI.

Our results was agreed with study of Basu RK et al. 2014 [8], which stated that the novel biomarker of AKI are not superior to RAI for prediction of sever AKI but it demonstrate equivalent performance for prediction of day 3 AKI. In our study there was statistically significant increase in RAI+ in comparison to RAI- with serum creatinine in critically ill children (P-value $<0.001$ ).

Our results was agreed with study of Shina Menon et al. 2016 [10], which stated that there was statistically significant increase in serum creatinine and renal angina fulfillment (RAI+) (P-value < 0.001).

In our study there was statistically significant decrease in RAI+ in comparison to RAI- with urine output in critically ill children.

Our results was agreed with study of Basu RK et al. 2014 [8], which stated that significant difference in pediatric illness severity (PRISM-II score which measured 
either by urine output or change in creatinine or the worth metric of two) between RAI+ and RAI-.

In our study there was statistically significant deference in sepsis as regards RAI+ in critically ill children (P-value $<0.028$ ).

Our results were agreed with study Shina Menon et al. 2016 [10], which state that approximately one in three patients (32.6\%) was RA+ within the first $12 \mathrm{~h}$ of PICU admission. These patients had a higher prevalence of sepsis.

In our study there was no statistically significant difference in demographic data regarding RAI. (Age, sex, height, weight) with day $3 \mathrm{AKI}$ in critically ill children.

Our study agreed with Basu RK et al. 2014 [8], but they found that younger age was independently associated with day $3 \mathrm{AKI}$ in critically ill children.

\section{Conclusion}

- uNGAL increase in critically ill children with AKI in pediatric ICU.

- Increase the rate of AKI in critically ill children with RAI+.

- Incorporation of UNGAL with RAI enhances the predictive ability of AKI in critically ill children.

\section{References}

1. Susantitaphong $P$, Cruz DN, Cerda J, Abulfaraj $M$, Alqahtani $F$, et al. World incidence of AKI: a metaanalysis. Clin J Am Soc Nephrol. 2013; 8: 1482-1493. Ref.: https://bit.ly/2Qr8cJB

2. Himmelfarb J, Joannidis M, Molitoris B, Schietz M, Okusa MD, et al. Evaluation and initial of acute kidney injury. Clin J Am Soc Nephrol. 2008; 3: 962-967. Ref.: https://bit.ly/2QwOETZ

3. Khwaja A. KDIGO clinical practice guidelines for acute kidney injury. Nephron Clin Pract. 2012; 120: c179-184. Ref.: https://bit.ly/2Ma1KrH

4. Devarajan P. Biomarkers for the early detection of acute kidney injury. Curr Opin Pediatr $2011 ; 23$ : 194-200. Ref.: https://bit.ly/2HETM5C

5. Meersch M, Schmidt C, Van Aken H, Rossaint J, Görlich D, et al. Validation of cell-cycle arrest biomarkers for acute kidney injury after pediatric cardiac surgery. PLoS One. 2014; 9: e110865. Ref.: https://bit.ly/2wIELPO

6. Meyer M, Fink C, Roeger S, Apfaltrer $P$, Haghi $D$, et al. Benefit of combining quantitative cardiac CT parameters with troponin I for predicting right ventricular dysfunction and adverse clinical events in patients with acute pulmonary embolism. Eur J Radiol. 2012; 81: 3294-3299. Ref.: https://bit.ly/2W8HawL

7. Zappitelli M, Parikh CR, Akcan-Arikan A, Washburn KK, Moffett BS, et al. Ascertainment and Epidemiology of Acute Kidney Injury Varies with Definition Interpretation. Clin J Am Soc Nephrol. 2008; 3: 948-954. Ref.: https://bit.ly/2MdHoxK

8. Basu RK, Wang Y, Wong HR, Chawla LS, Wheeler DS, et al. Incorporation of biomarkers with the renal angina index for prediction of severe AKI in critically ill children. Clin J Am Soc Nephrol. 2014; 9: 654-662. Ref.: https://bit.ly/217sAvh

9. Bolignano D, Basile G, Parisi P, Coppolino G, Nicocia G, et al. Increased plasma neutrophil gelatinaseassociated lipocalin levels predict mortality in elderly patients with chronic heart failure. Rejuvenation Res. 2009; 12: 7-14. Ref.: https://bit.ly/2Ww2ifz

10. Menon S, Goldstein SL, Mottes T, Fei L, Kaddourah A, et al. Urinary biomarker incorporation into the renal angina index early in intensive care unit admission optimizes acute kidney injury prediction in critically ill children: a prospective cohort study. 2016; 31: 586-594. Ref.: https://bit.ly/2wINXUq 